\title{
1 Characterization of a Micro-hydrokinetic Turbine in Close Proximity to
}

2 the Free Surface

3 JACOB RIGLIN, Ph.D. Student, P.C. Rossin College of Engineering and Applied Science,

4 Lehigh University, Bethlehem, PA, 18015, USA

5 Email:jar611@lehigh.edu

6 W. CHRIS SCHLEICHER, Postdoctoral Research Associate, P.C. Rossin College of

7 Engineering and Applied Science, Lehigh University, Bethlehem, PA, 18015, USA

$8 \quad$ Email:wcs211@lehigh.edu

I-HAN LIU, Ph.D. Student, P.C. Rossin College of Engineering and Applied Science, Lehigh

University, Bethlehem, PA, 18015, USA

Email:ihl211@lehigh.edu

ALPARSLAN OZTEKIN, Professor, P.C. Rossin College of Engineering and Applied

Science, Lehigh University, Bethlehem, PA, 18015, USA

Email:alo2@lehigh.edu

\section{ABSTRACT}

Predicting hydrokinetic turbine power generation is difficult due to complex geometry, highly turbulent conditions, and difficulty capturing the transient interface existing between air and water.A threedimensional finite volume solver was used to capture the effects resulting from free surface interaction with the aid of a Volume of Fluid(VOF) multiphase solver.Depths from free surface level to blade tip with corresponding Froude numbers of $0.71,0.92,1.04$, and 1.31 were modelled specifically to capture the transition from subcritical to supercritical flow conditions.A sharp decrease in performance was observed at the critical Froude number $(\mathrm{Fr}=1.0)$. Results at subcritical conditions showed acceptable agreement with previously published single phase results where the turbine is assumed to be operating in aninfinite medium.At subcritical conditions, the propeller-based turbine studied was compared to numerical and experimental results obtained for a traditional marine current turbine (MCT).As the flow became critical, a $32.2 \%$ decrease in the power coefficient was predicted and significant wake-free surface interaction was observed.

Keywords: Micro-hydrokinetic; Turbine Performance; Turbulence; Computational Fluid Dynamics; Volume of Fluid; Free Surface Interaction 


\section{Characterization ofaMicro-hydrokinetic Turbine in Close Proximity to 32 the Free Surface}

\section{Introduction}

Renewable energy solutions such as wind, solar, and hydro power are sought to minimize carbon emissions that adversely affect global climate. Horizontal axis wind turbines (HAWT) are a subset of turbomachinery that extract energy from free-flowing wind using carefully designed rotors. A similar concept is applied in tidal and current flows, where water is the acting fluid instead of air.Both kinetic applications are bounded by a theoretical limit, known as the Betz limit,which dictates the maximum energy that may be extracted from a free-flowing fluid. This limit has been determined to be $59.3 \%$ of the available kinetic energy within the medium.An optimal hydraulic design that maximizes power output is crucial. Mukherji et al. (2011) .and Kolekar et al. (2011, 2013)designed and optimized a horizontal axis hydrokinetic turbine using blade element momentum (BEM) theory and computational fluid dynamics (CFD) analysis.Geometric parameters, such as blade angle, number of blades, and chord length, were optimized resulting in $12 \mathrm{~kW}$ of mechanical power produced.Riglin et al. (2013) and Schleicher et al. $(2013,2014)$ designed a portable turbine that can produce $500 \mathrm{~W}$ of continuous power.A turbine design introduced by Schleicher $e t$ al.(2014, 2015)incorporated both propeller-based structure and a higher value of solidity comparable to that observed in the work conducted by Mukherji et al. (2011), Kolekar et al.(2011), and other traditional work conducted involving hydrokinetic and tidal turbine designs. Their turbines yielded apower coefficientof 0.43 that is about $73 \%$ of the available power based on the Betz limit.Bai et al.(2012) conducted numerical simulations of a $0.8 \mathrm{~m}$ diameter, marine current turbine (MCT) that yields a power coefficientof approximately 0.46 at the best efficiency point (BEP). Experimental results obtained by Bahaj et al.(2007)were measured using a $2.4 \mathrm{~m}$ by $1.2 \mathrm{~m}$ cavitation tunnel. Numerical results obtained by Bai et al. (2012) yielded accuracy corresponding to less than $1.0 \%$ error between experimental and numerical results for the tested MCT. 
Free surface interaction with submerged objects has been investigated extensively for

cylinders. The dynamics of the flow around submerged cylinders at varying depths was characterized by Reichl et al.(2005). The drag and lift coefficients were documented to have significantly lower average values than the values obtained at conditions of extreme submersion where the free surface has no interaction with the object. Malavasi et al.(2007) conducted experiments on a prism submerged obstructing flow in a channel. In both studies, when the obstruction was near the free surface, a breakdown in vortex shedding was experienced and an asymmetric, jet-like flow was observed. Expanding work conducted by Reichl et al.(2005) and Malavasi et al.(2007) to hydrokinetic turbines involves the addition of a circumferential velocity component corresponding to turbine rotation rate.

The free surface can result in substantially adverse conditions, including vibration and performance reductions, when hydrokinetic turbines are operated in close proximity. The work done by Schleicheret al. $(2013,2015)$ and Riglin et al. $(2013,2014,2015)$ for hydrokinetic river application assumes that the depth of the river is variable in both time and space and that the depth is a critical design limitation necessary for broad application. As determined through data provided by the United States Geological Survey (USGS) regarding river freestream velocities and average depths, many rivers in the United States have approximately two to three meters of average depth or less. Additionally, only $51.0 \%$ of rivers have velocities suitable for hydrokinetic turbine operation assuming a velocity range from $0.75 \mathrm{~m} / \mathrm{s}$ to $2.5 \mathrm{~m} / \mathrm{s}$ (U.S. Geological Survey, 2014). Wide spread application of a portable device is not only limited by velocity but river depth. Determining the depth necessary to guarantee suitable turbine operation and performance with negligible free surface effects is crucial for river applications. Whelan et al.(2009) provides a simplified, two-dimensional analysis of free surface and blockage effects involving tidal turbines in channels. BEM theory was applied for low Froude numbers of 0.22 and yielded acceptable accuracy and agreement with experimental results. Bai et al.(2014) conducted simulations incorporating the Volume of Fluid(VOF) model to determine losses resulting in near-free surface turbine operation. A depth of 0.55 turbine diameters was used with a freestream velocity of $1.73 \mathrm{~m} / \mathrm{s}$, 
corresponding to a Froude number of 0.83. Bai et al.(2014) reported a minor drop of approximately 1-2 percent of the BEP determined by Bahaj et al.(2007) and Bai et al.(2012). Although the free surface effects were modeled, varying Froude numbers and the characterization of the turbine at conditions reflecting critical flowwere not modeled. The blade proposed by Schleicher et al.(2015) that was used in the present study is depicted in Figure 1(a). Figure 1(b) shows the blade design that was used in the experimental study conducted by Bahaj et al. (2007) and the numerical study presented by Bai et al. (2014). Kolekar and Banerjee (2015) have also investigated turbine performance near the vicinity of the free surface. The present study highlights the efforts of understanding the adverse effects resulting from operation of a hydrokinetic turbine in close proximity to the free surface, or air-water interface between the flowing river and the atmosphere. The impact of Froude number, at both subcritical and supercritical values, on turbine operation was investigated numerically based on the propeller-based design proposed by Schleicher et al.(2013, 2014, 2015).

(a)

(b)
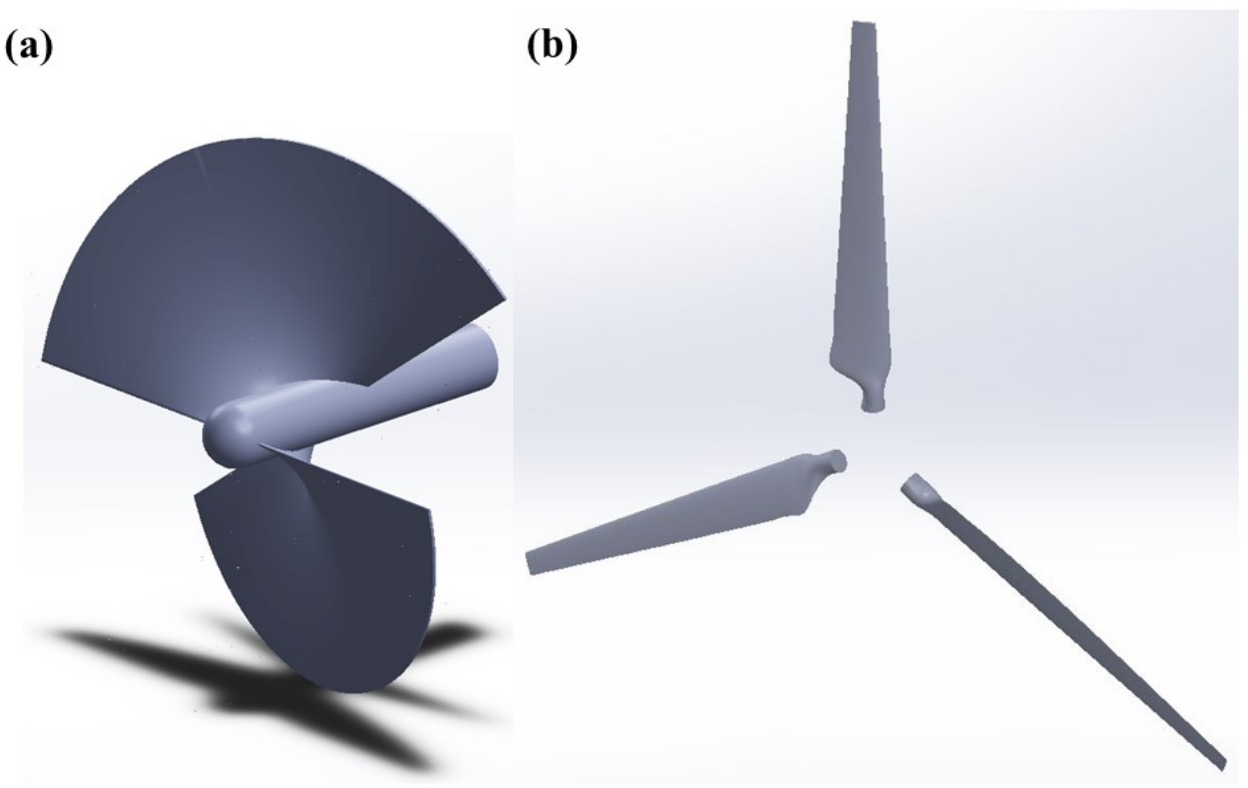

Figure 1. (a) Blade design documented by Schleicher et al.(2015), and (b) blade design used in experimental and numerical investigations conducted by Bai et al. (2014) and Bahaj et al. (2007). 


\subsection{Turbine design and river domain}

The propeller-based turbine used in the present study was designed using a bottom-up

approach. Geometric parameters were determined based on preliminary goals of power, power coefficient, and freestream velocity specified a priori (Schleicher, 2015).Schleicher et al.(2015)proposed a high solidity $(\sigma)$ turbine, as shown in Figure 2.The tip diameter $\left(D_{t}\right)$, hub diameter $\left(D_{h}\right)$, mean diameter $\left(D_{m}\right)$ and wrap angle $(\Delta \theta)$ are depicted in Figure 2(a). The meridional blade length $(\Delta m)$ and the relative blade angle $\left(\beta^{\prime}\right)$ are displayed inFigure 2(b).

112 Schleicher et al.(2015)provided the step-by-step design approach while Cebrián et al.(2013)provided a relation to the relative blade angle used in the design process. Solidity $(\sigma)$ and blade number $\left(Z_{B}\right)$ were chosen based on designer experience. All input and output Table 1.

(a)

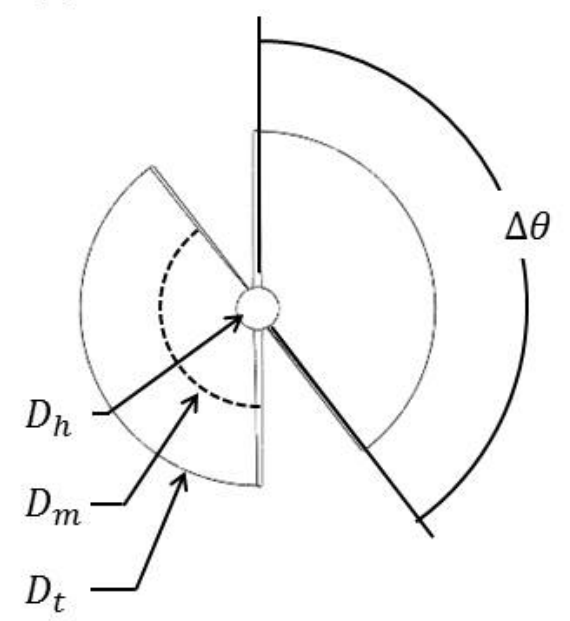

(b)

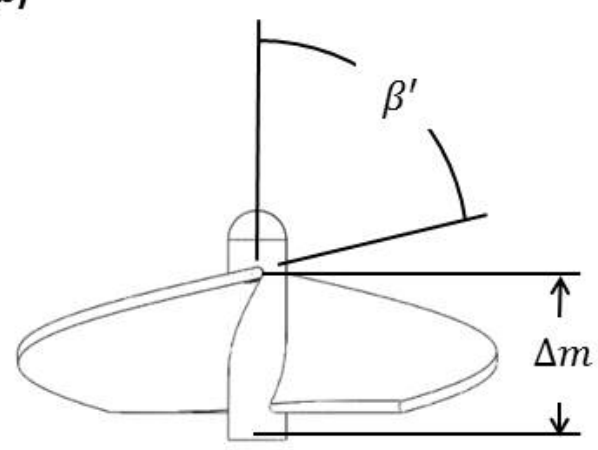

117

Figure 2. Propeller based turbine design (a) front and (b) top view.

119 Table 1. Basic turbine design parameters

\begin{tabular}{clccll}
\hline \multicolumn{5}{c}{ Input Design Variables } \\
\hline$P$ & 500 & $(\mathrm{~W})$ & $\omega$ & 15.708 & $\left(\mathrm{~s}^{-1}\right)$ \\
$C_{P}$ & 0.4 & \multicolumn{5}{c}{$Z_{B}$} & 2 \\
$U_{\infty}$ & 2.25 & $\left(\mathrm{~ms}^{-1}\right)$ & $\sigma$ & 0.83 \\
\hline \multicolumn{5}{c}{ Output Design Variables } \\
\hline$D_{t}$ & 0.533 & $(\mathrm{~m})$ & $\Delta \theta$ & 142.29 & $\left(^{\circ}\right)$
\end{tabular}


$\begin{array}{llllll}D_{h} & 0.063 & (\mathrm{~m}) & \beta & 52.58 & \left(^{\circ}\right)\end{array}$

$\begin{array}{lllllll}D_{m} & 0.374 & (\mathrm{~m}) & \Delta m & 0.1488 & (\mathrm{~m})\end{array}$

The river domain design that was implemented is shown in Figure 3. Turbine and free

surface level is depicted in Figure 3(a) such that the free surface is set to a distance of $6.86 D_{t}$

from the channel bottom for each design. The turbine depth $(d)$ is the distance between the minimize the effect it had on the computed flow field.

(a)

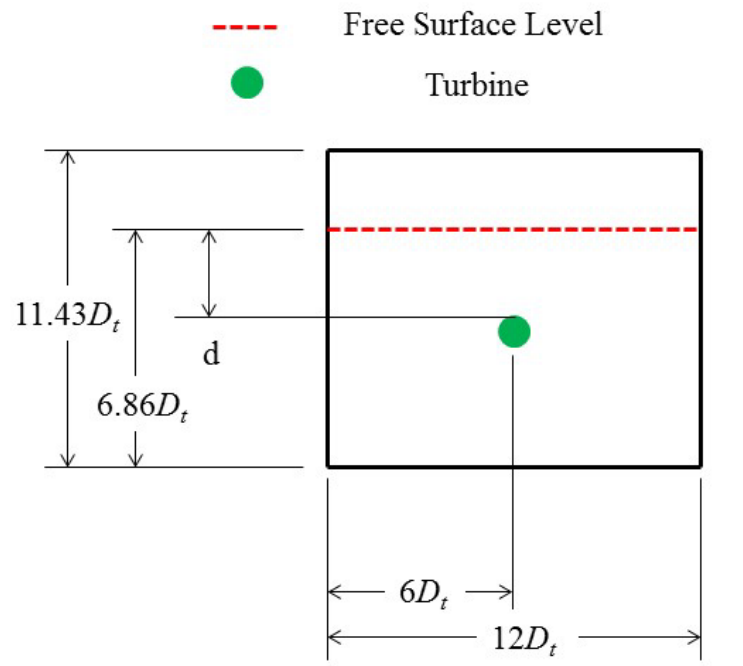

(b)

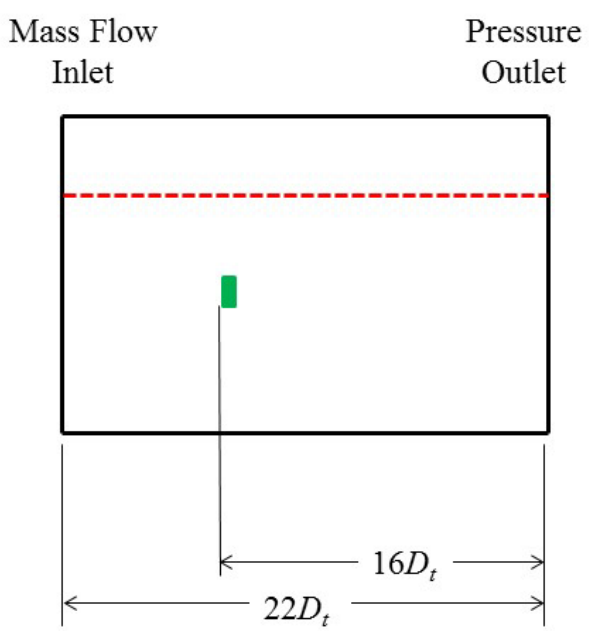

128

Figure 3. Front view (a) and side view (b) of the river domain with dimensions based on turbine diameter $\left(D_{t}\right)$.

Theflow field near the turbine within the river can be characterized using Reynolds number andFroude number.

$$
R e=\frac{\rho U_{\infty} D_{t}}{\mu} \quad F r=\frac{U_{\infty}}{\sqrt{g d}}
$$

Here $R e$ is the Reynolds number, $\rho$ is the fluid density, $U_{\infty}$ is the freestream velocity magnitude experienced by the turbine, and $\mu$ is the dynamic viscosity. $F r$ is the Froude number, $d$ is the depth, and $g$ is gravitational acceleration. All simulations are conducted for a Reynolds number of approximately 1.19 million. 
Free surface effects will vary depending on the turbulence experienced within the

139 river, both turbulence generated during channel flow as well as turbulence produced by the

140 turbine itself. Froude number will also play a critical role in the development of the transient

141 free surface level.The present study focuses only on a single Reynolds number.However, for a

142 fixed value of $F r$ the effect of the free surface at a higherReynolds number will be more

143 noticeable.

144 Key locations within the computational domain used are shown in Figure 4. The cross

145 sectional volume discretization of the central, refined portion of the domain is shown in

146 Figure 4(a). The grid structure formed along the turbine blade, Figure 4(b), is positioned such

147 that the cells have an aspect ratio value close to one.The entire computational domain,

148 containing turbine and river subdomains, was discretized with structured, hexahedral cells.

149 Three separate domains were used, each consisting of the same dimensions of $11.43 D_{t}$ in

150 height, $12 D_{t}$ in width, and $22 D_{t}$ in length with $16 D_{t}$ from the leading edge of the turbine

151 blades to the outlet. Additionally, each domain mesh consisted of the same number of cells,

152 which was approximately 11.6 million. Schleicher et al. (2015) conducted spatial

153 convergence analysis on the present blade design and determined that a mesh size of 6.0

154 million resulted in a Grid Convergence Index (GCI) error of 3.1\% for torque production using

155 Richardson extrapolation proposed by Roach et al.(1993). The grid in the present design is

156 refined further beyond the level that was used in previous studies. Domains differed in that

157 the turbine region was oriented differently based upon depth. Depths of $0.3048 \mathrm{~m}, 0.4572 \mathrm{~m}$,

$158 \quad 0.6096 \mathrm{~m}$, and $1.2192 \mathrm{~m}$ were used corresponding to the Froude numberof 1.31, 1.04, 0.92,

159 and 0.71 , respectively. The present mesh structure employed wall functions to determine flow

160 separation and provided average $y+$ values of approximately 30.0. 
(a)
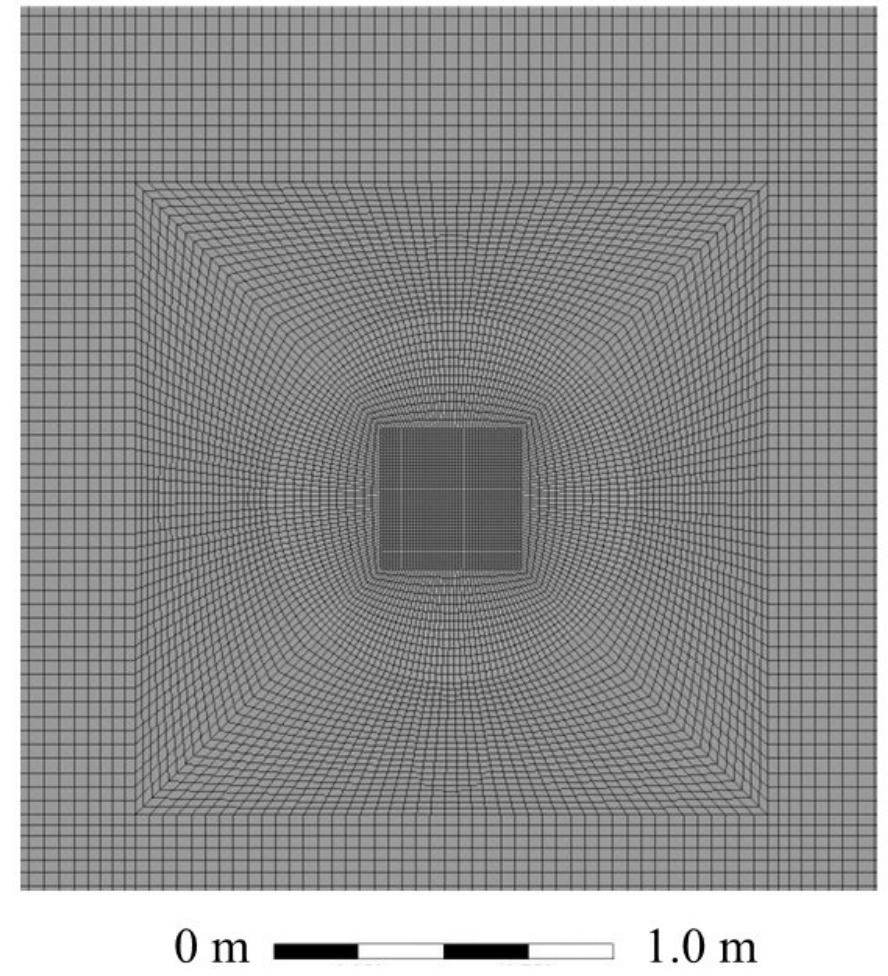

(b)

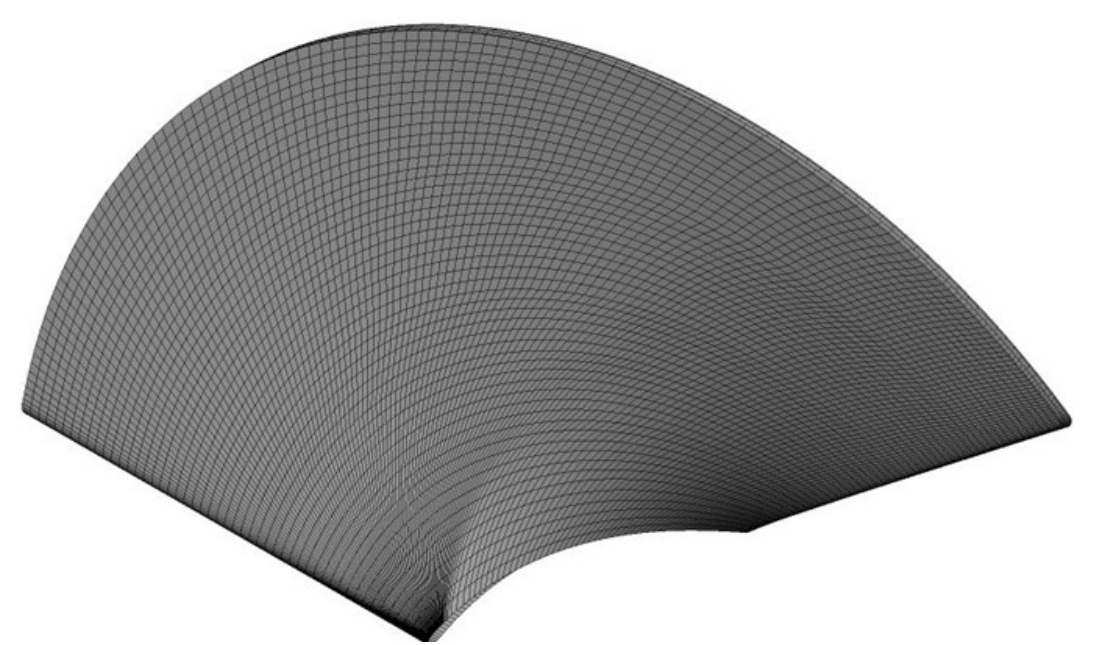

Figure 4. (a) Center of the computational domain for $\mathrm{Fr}=0.70$ and (b) grid along a single turbine blade.

\subsection{Turbulence and flow modelling}

165 Transient simulations were conducted using the Pressure Implicit with Splitting of

166 Operators(PISO)pressure-velocity coupling solver. Steady-state solutions are only achievable

167 for VOF, multiphase modeling when the solution is independent of the initial condition. To

168 obtain the final solution and ensure the stability of the calculations, transient analysis was 
necessary for flow-modeling purposes. A sliding mesh was incorporated for the turbine domain, set at a rotational value corresponding to the turbine rotation. Grid to grid interface interpolation was conducted between the turbine domain and the river domain. The free surface was modeled directly using the implicit VOF model. Noh and Woodward (1976) developed the Simple Line Interface Calculation (SLIC) as the first formulation for tracking an interface that exists between two fluids. Hirt and Nichols (1981) expanded upon tracking the fluid interface by formulating the VOF model. The implicit VOF formulation of mass transfer, and momentum are provided in Equations (2), (3), and (4).

$$
\begin{gathered}
\frac{1}{\rho_{q}}\left[\frac{\partial}{\partial t}\left(\alpha_{q} \rho_{q}\right)+\frac{\partial}{\partial x_{i}}\left(\alpha_{q} \rho_{q} U_{q, i}\right)=M_{\alpha_{q}}+\sum_{p=1}^{n}\left(\dot{m}_{p q}-\dot{m}_{q p}\right)\right] \\
\frac{\alpha_{q}^{n+1} \rho_{q}^{n+1}-\alpha_{q}^{n} \rho_{q}^{n}}{\Delta t} V_{c}+\sum_{f}\left(\rho_{q} \bar{U}_{f}^{n+1} \alpha_{q, f}^{n+1}\right)=\left[\sum_{p=1}^{n}\left(\dot{m}_{p q}-\dot{m}_{q p}\right)+M_{\alpha_{q}}\right] V_{c} \\
\frac{\partial}{\partial t}\left(\rho U_{i}\right)+\frac{\partial}{\partial x_{j}}\left(\rho U_{j} U_{i}\right)=-\frac{\partial}{\partial x_{i}} P+\frac{\partial}{\partial x_{j}}\left(\mu\left(\frac{\partial U_{j}}{\partial x_{i}}+\frac{\partial U_{i}}{\partial x_{j}}\right)\right)+\rho g_{i}+F_{i}
\end{gathered}
$$

where $V_{c}=V_{q}+V_{p}$ is the cell volume, $V_{q}$ is the volume of the primary phase, $V_{p}$ is the volume of the secondary phase, and $M$ is the mass source term. In the current study, the primary phase was specified as air while the secondary phase was water. The term $\alpha_{q}=$ $V_{q} / V_{c}$ indicates the volume fraction, locally within the cell, of the primary phase and it satisfies $\sum_{q=1}^{n} \alpha_{q}=1$. The volume fraction of the $\mathrm{q}_{\mathrm{th}}$ phase is represented as $\alpha_{q}$ and is bounded between zero and one. The tensor velocity is provided as $U_{i}$ and the pressure is specified by the term $P$. The terms $\dot{m}_{q p}$ and $\dot{m}_{p q}$ represent the mass transfer from phase $q$ to phase $p$ and vice versa. The volume flux through the face of each cell, based on the normal velocity, is provided by $U_{f}$.

Turbulence existing in the flow field was modeled using Menter's $k$ - $\omega$ Shear Stress Transport ( $k$ - $\omega S S T)$ (Menter, 1993; Menter, 1994)two-equation eddy-viscosity model. This model offers improved prediction of adverse pressure gradients in the near wall region as compared to the standard $k-\omega$ and $k-\varepsilon$ models by incorporating Bradshaw's observation that turbulent shear stress is proportional to the turbulent kinetic energy in the wake region of the boundary layer (Wilcox, 2010). The $k$ - $\omega$ SST model imitates the $k$ - $\omega$ model at near wall 
regions, allowing for flow separation to be accurately predicted. At regions far away from the wall, $k$ - $\omega$ SST model performs like the $k-\varepsilon$ model and accurately models the wake. The $k-\omega$ SST model utilizes the strengths of the $k$ - $\omega$ model and the $k-\varepsilon$ model ensuring that both the turbine performance characteristics and wake profiles produced are accurate. Formulations of kinematic eddy viscosity, turbulent kinetic energy, and specific dissipation rate are included in Equations (5), (6), and (7) below:

$$
\begin{gathered}
v_{T}=\frac{\alpha_{1} k}{\max \left(\alpha_{1} \omega, S F_{2}\right)} \\
\frac{\partial k}{\partial t}+U_{j} \frac{\partial k}{\partial x_{j}}=\tau_{i j} \frac{\partial U_{i}}{\partial x_{j}}-\beta^{*} k \omega+\frac{\partial}{\partial x_{j}}\left[\left(v+\sigma_{k} v_{T}\right) \frac{\partial k}{\partial x_{j}}\right] \\
\frac{\partial \omega}{\partial t}+U_{j} \frac{\partial \omega}{\partial x_{j}}=\alpha S^{2}-\beta \omega^{2}+\frac{\partial}{\partial x_{j}}\left[\left(v+\sigma_{\omega} v_{T}\right) \frac{\partial \omega}{\partial x_{j}}\right]+2\left(1-F_{1}\right) \sigma_{\omega 2} \frac{1}{\omega} \frac{\partial k}{\partial x_{i}} \frac{\partial \omega}{\partial x_{i}}
\end{gathered}
$$

where $v_{T}$ is the turbulent eddy viscosity, $v$ is the kinematic viscosity, $k$ is the turbulent kinetic energy, $\omega$ is the specific dissipation rate, $\alpha_{1}$ is a closure coefficient, $S$ is the mean rate-of-strain tensor, and $F_{1}$ and $F_{2}$ are blending functions. The details of the $k-\omega$ SST turbulence model are documented in Wilcox (Wilcox, 2010).

In the current open channel simulation, numerical reflection of waves is caused by the outlet and results in numerical instability. Numerical Beach Treatment was applied to the inlet of the river domain. The numerical beach acts as a damping sink and prevents the numerical reflections that may be generated near the pressure outlet boundary. Equations implementing the damping are listed below (Park et al., 1999; Zwart et al. 2007):

$$
\begin{array}{cr}
\varphi=c\left(\frac{1}{2} \rho\left|U_{y}\right| U_{y}\right) g(y) g(z) & \\
r_{z}=\frac{z-z_{s}}{z_{e}-z_{s}} & r_{y}=\frac{y-y_{f s}}{y_{b}-y f_{s}} \\
g(z)=\left(r_{z}\right)^{2} & g(y)=1-r_{y}
\end{array}
$$

Here $\varphi$ is the momentum sink term, cis the damping resistance, and $g(y)$ and $g(z)$ are the damping functions in the $y$-direction and $z$-direction. Damping functions are composed of two independent, dimensionless scaling factors, $r_{z}$ and $r_{y}$. The points at which the damping zone begin and end are denoted by $z_{s}$ and $z_{e}$. The position of the free surface along the direction 
corresponding to the gravity normal vector is specified through $y_{f s}$ while the channel bottom 221 is $y_{b}$.

A PISO Pressure-Velocity coupled solver was incorporated with pressure correction produced through the use of the Pressure Staggering Option(PRESTO!) scheme. Momentum, turbulent kinetic energy, and turbulent dissipation rate were all modelled with Second-order Upwind schemes. To capture the time-averaged interface between the air phase and the water phase, the Modified High Resolution Capturing (HRIC) scheme was used in determining the volume fraction. The transient effects were captured with a Second-order Upwind scheme while using a time step of $0.001 \mathrm{~s}$. The boundary conditions imposed on the velocity and the pressure fields were: a noslip and no-penetration wall on the river bed, sides, and top of the domain, constant mass flow rate at the inlet, and a zero pressure condition at the outlet. The mass flow rate was specified at the inlet for each phase. A value of $52570.2 \mathrm{~kg} / \mathrm{s}$ was specified for the water phase (yielding an approximatefreestream velocity of $2.25 \mathrm{~m} / \mathrm{s}$ ) and a value of $0.01 \mathrm{~kg} / \mathrm{s}$ was applied to the air region to ensure an air flow of approximately zero.The turbulence intensity was specified as $2.233 \%$ at both the inlet and outlet of the domain. The outlet utilized a zero gradient condition for velocity at the outlet with constant pressure. Open channel boundary conditions were applied at both the inlet and outlet indicating both the free surface level within the channel as well as the depth of the channel from the origin. The free surface level and channel depth were specified based on the orientation of the fluid geometry and corresponding Froude number. A damping resistance, $c$, of 10 1/m was applied to each case.A sliding mesh with a three general grid interfaces (GGI) between the turbine domain and the river domain was incorporated to model the rotation of the turbine. The Courant-Friederichs-

243 Lewy condition, provided below, was applied to ensure the stability and accuracy of the 244 computational results.

$$
C u=\frac{U_{\infty} \Delta t}{\Delta z}<C u_{\max }
$$


where $C u$ is the Courant number, $\Delta \mathrm{z}$ is the cell size in the flow direction, $\Delta t$ is the time step

247 increment, and $C u_{\max }$ isthe maximum Courant number. The maximum Courant number

248 necessary for stability is 1.0.With the time step selected, for the given set of flow conditions

249 and the grid size, the average Courant number of the computational domain was

250 approximately 0.2 . The time step selected also allowed for the turbine domain to rotate

251 exactly $0.9^{\circ}$ at each step, allowing for transient effects of the turbine and wake to be captured

252 with accuracy and stability. The convergence criteria for momentum, continuity, turbulence

253 parameters, and the volume fraction of relative residuals were set to value of $10^{-4}$.

254

255

256

\subsection{Validation of the model and the numerical method}

Experimental data provided by Malavasi and Guadagnini (2007)on interaction between a rectangular cylinder and the free surface was compared to numerical predictions using the VOF model(Liu et al.,2015). Figure 5 shows the geometric orientation of the rectangular cylinder in the open channel. A $5 \mathrm{~m}$ long channel was modelled with a crosssectional width, $B$, of $0.5 \mathrm{~m}$. The rectangular cylinder was fully submerged at a depth of $h^{*}$, from free surface to the most submerged surface of the cylinder. Parameters along with the corresponding descriptions and values are included inTable 2 . The simulations conducted had a Reynolds number of $2.02 \times 10^{4}, h^{*}=\left(h-h_{b}\right) / s=4$, and an elevation to cylindrical cross sectional width ratio of $h_{b} / s=2.33$.

(a)

(b)

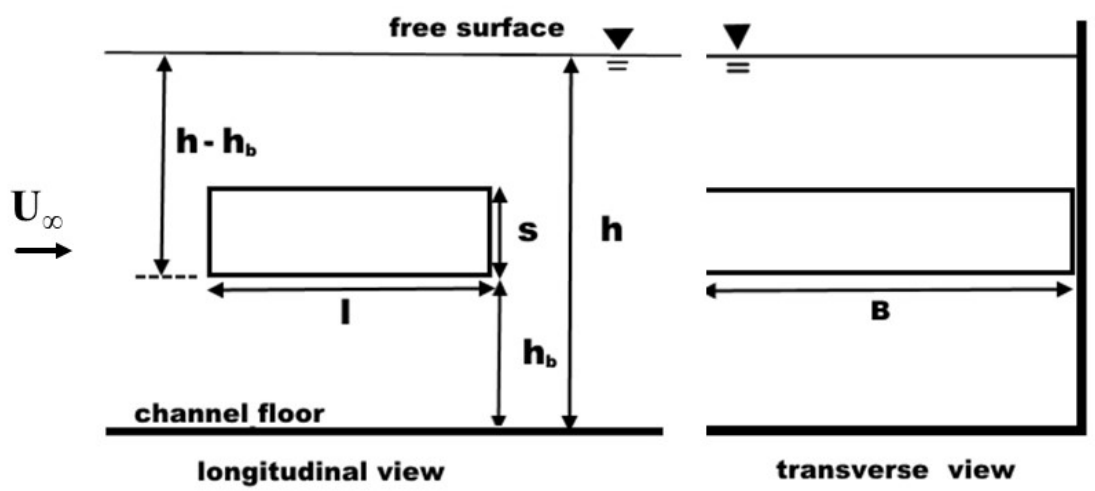

Figure 5. Channel orientation in both (a) the longitudinal view and (b) the transverse view(Liu et al., 2015). 
268 Table 2. Parameter values used for the validation

\begin{tabular}{llll}
\hline \multicolumn{2}{l}{ Parameter Values } & & \\
\hline$l$ & cylinder cross-section length & 0.18 & $(\mathrm{~m})$ \\
$s$ & cylinder cross-section width & 0.06 & $(\mathrm{~m})$ \\
$h$ & water depth & 0.3798 & $(\mathrm{~m})$ \\
$h_{b}$ & cylinder elevation above channel floor & 0.1398 & $(\mathrm{~m})$ \\
$h^{*} \quad$ non-dimensional depth & 4 & \\
$B$ & channel width & 0.5 & $(\mathrm{~m})$ \\
$\mathrm{U}_{\infty}$ & upstream velocity & 0.334 & $\left(\mathrm{~m} \mathrm{~s}^{-1}\right)$ \\
\hline
\end{tabular}

269

270

271

272

273

274

275

276

277

278

279

280

281

282

283 284

Three terms were used in the quantitative validation of the VOF model implemented:

the drag coefficient, $C_{D}=F_{D} / \frac{1}{2} \rho U_{\infty}{ }^{2} A$, the lift coefficient, $C_{L}=F_{L} / \frac{1}{2} \rho U_{\infty}{ }^{2} A$, and the Strouhal number, $S t=f s / U_{\infty}$. The area is represented by $A$, the drag and lift forces are represented by $F_{D}$ and $F_{L}$, and the cylinder cross-section width and shedding frequency are given as $s$ and $f$.The mean drag coefficient, mean lift coefficient, and Strouhal number observed in the validation simulation were $1.69,-0.67$, and 0.17 , respectively. These predicted values all had relative errors of $6.1 \%$ or less when compared to results obtained by Malavasi and Guadagnini (2007)with nearly matching Strouhal numbers for both experimental and numerical results. The minimal relative errors matching Strouhal numbers indicate that the simulation results match well with the experimental result and provide confidence in the modelling and methods used to capture the transient effects occurring between the turbine unit and the free surface.
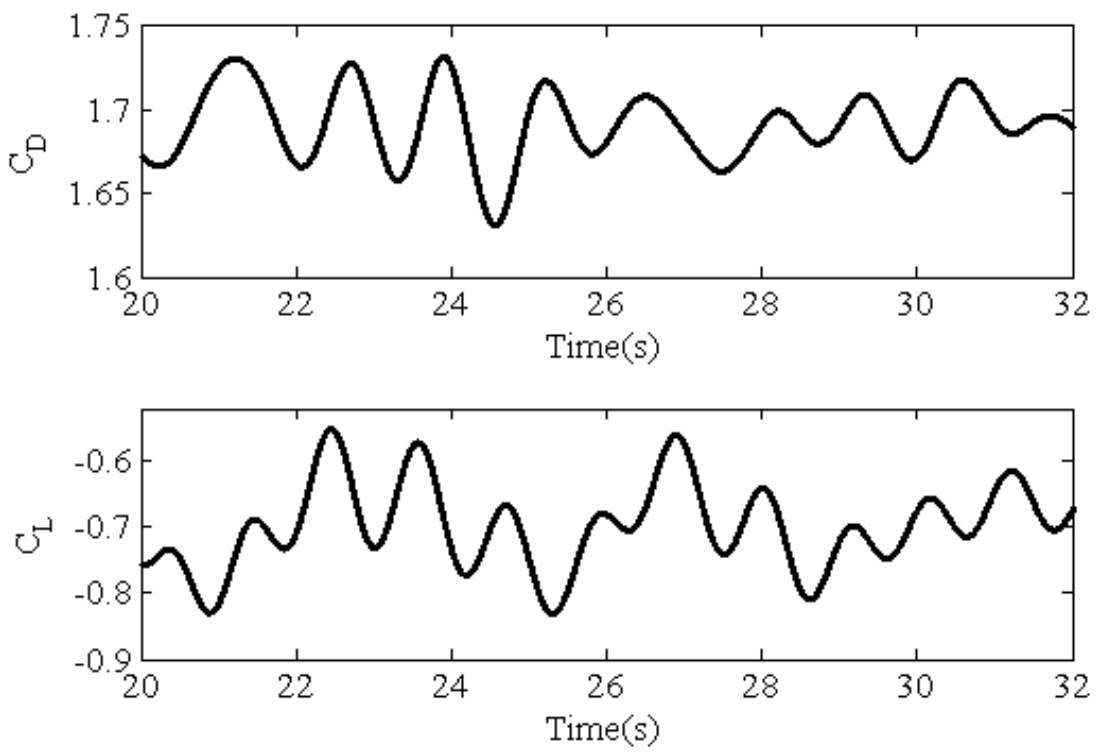

Figure 6. Values of coefficient of (a) drag and (b) lift with respect to flow time (Liu et al,. 2015). 


\section{Results}

\subsection{Performance Characteristics}

The performance of the turbineunit was characterized using the non-dimensional parameters of tip speed ratio $(\lambda)$, power coefficient $\left(C_{P}\right)$, and thrust coefficient $\left(C_{T}\right)$, shown in equation (12).

where $\dot{W}$ is the power produced by the turbine, and $T$ is the thrust. Figure 7(a) and Figure 7(b) show the power coefficient and the thrust coefficient as a function of the Froude number compared to turbine operation in a single phase, infinite medium. Figure 7(a) also shows the BEP of the traditional hydrokinetic turbine used by Bahaj et al. (2007) which was obtained through numerical work and validated with experiments, along with the corresponding results of the multiphase simulation conducted by Bai et al.(2014). The work conducted by Bai et al.

300 (2014) yields an approximate $4 \%$ drop in power at $F r=0.82$. Based on the definition of the

301 Froude number, as the depth of the turbine approaches infinity, the performance of the unit

302 should approach an asymptotic value corresponding to that in an infinite medium. As the depth of the unit being modelled approaches zero, the value of Froude number will approach infinity. The multiphase simulation results presented in Figure $7 \mathrm{a}$ indicates that power produced at subcritical Froude numbers of 0.92 and 0.71 has less than $5 \%$ deviation when compared against the single phase results produced by Schleicher et al. (2015). The plot of the power coefficient suggests that near the critical Froude number $(\mathrm{Fr}=1)$, a substantial drop in power is experienced. Between Froude numbers of 0.92 and 1.04, the power coefficient drops from a value of approximately 0.45 to 0.30 , representing a $33 \%$ reduction in power under the same freestream flow conditions. As indicated by Figure 7(b), the thrust coefficient

311 experiences a similar reduction; however the drop experienced by the turbine thrust is 
312 significantly more gradual despite predominantly occurring near the critical Froude number.

313 The performance results obtained suggest that the turbine must be submerged to a depth that

314 provides a subcritical Froude number in relation to the freestream velocity of turbine

315 operation.

(a)

- Multiphase-Free Surface

\ Bai et al. (2014)

-----Single Phase, Schleicher et al. (2015)

......... Bahaj et al. (2007)

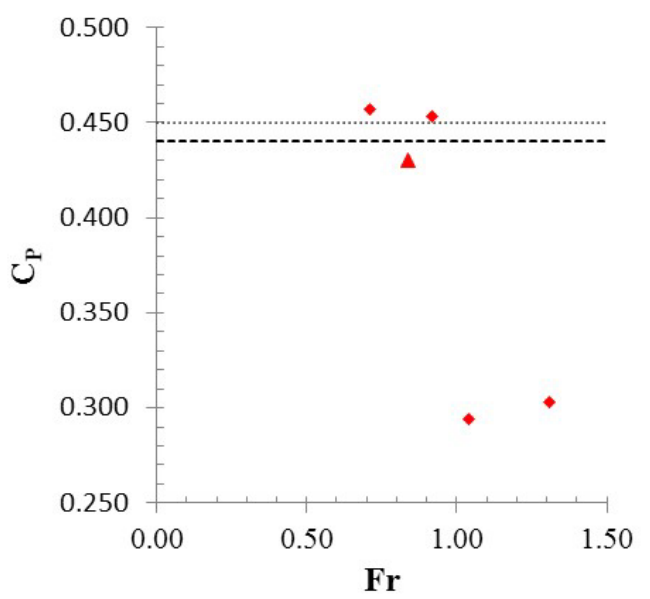

(b)

- Multiphase-Free Surface

--- Single Phase, Schleicher et al. (2015)

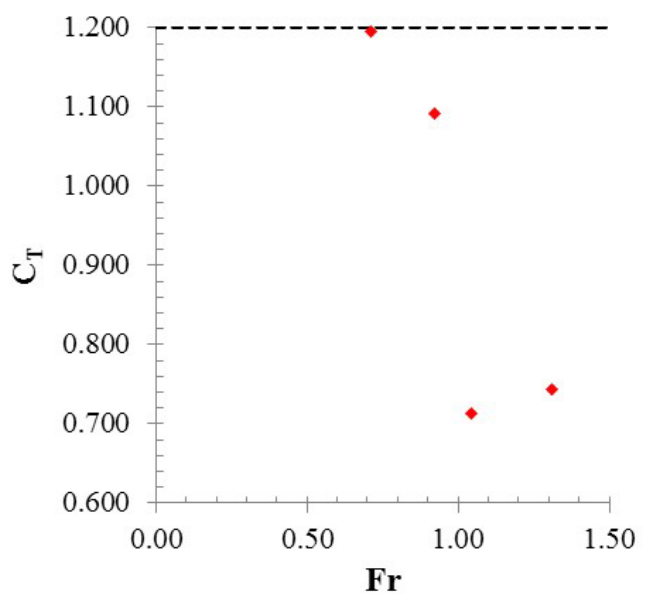

316

317

318

319

320

321

322

323

324

325

326

327

328

Figure 7. (a) Power coefficient and (b) thrust coefficient as a function of $F r$.

A comparison of the power coefficient as a function of tip speed ratio

between both propeller-based and traditional, hydrofoil blade design is displayed in Figure 8.

Both designs produce curves that peak around a power coefficient of approximately 0.44 to

0.45 . The primary disparity between the curves generated by numerical results from Shleicher et al. and experimental results from Bahaj et al. is that the tip speed ratio existing for the propeller-based turbine design has a much smaller range, occurring at lower values, than the operating conditions of the traditional design. The point at which peak performance occurs for the propeller-based design is approximately a third of the traditional design. Multiphase results, shown in red below, indicate a sufficient drop in peak power production, at a Froude number of approximately one. The numerical results presented by Bai et al.(2014) show only 
Froude number.

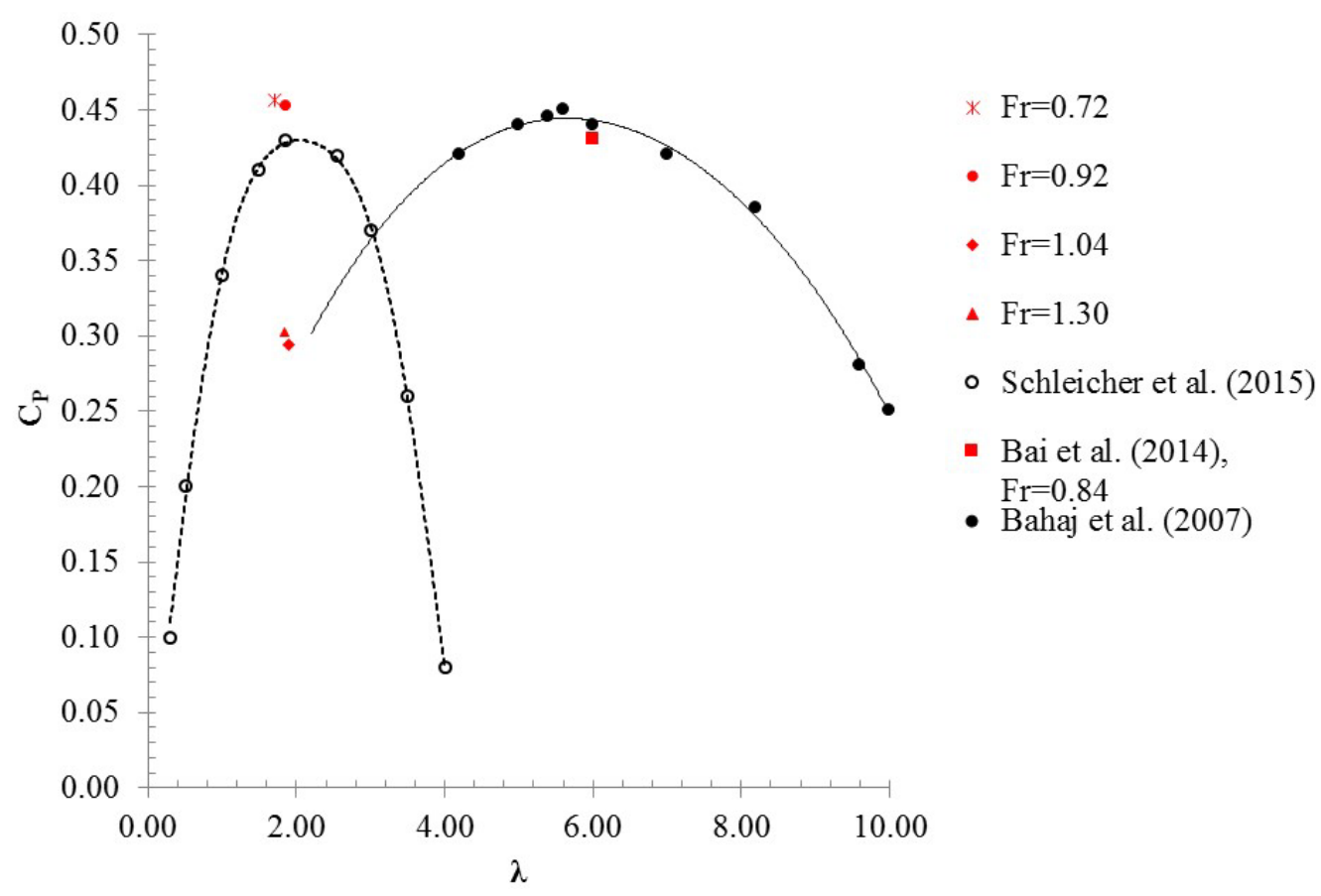

331

332

333

334

Figure 8. Power coefficient as a function of tip speed ratio for both traditional and propellerbased hydrokinetic turbines studied (Schleicher et al., 2015; Bahaj et al., 2007; Bai et al., 2014).

\subsection{Wake Characteristics}

Pictured in Figure 9 and Figure 10 are the flow-field results for the single and multiphase simulations along the vertical plane, located in the center of each respective computational domains, for a free-stream velocity of $2.25 \mathrm{~m} / \mathrm{s}$ and constant rotation rate of $150 \mathrm{rpm}$. The method of normalization for both velocity and vorticity is provided in equation (13).

$$
\widetilde{U}=\frac{\left|U_{i}\right|}{U_{\infty}} \quad \widetilde{\omega}=\frac{\left|\epsilon_{i j k} \frac{\partial U_{k}}{x_{j}}\right|}{\omega}
$$

Trailing tip vortices can be observed based on the normalized velocity gradients within Figure 9(a) and the regions of intense normalized vorticity (values exceeding one) in Figure 9(b). Figure 9 represents a base, single phase analysis of the propeller-based turbine operating in what may be considered an infinite medium, with no effects from boundary layers, and in 
346 which the flow is subcritical. Multiphase results representing operation at Froude numbers of

3470.92 and 1.31 are displayed in Figure 10.Transition from supercritical flow to subcritical flow

348 occurs from Figure 10(a) and Figure 10(b) to Figure 10(c) and Figure 10(d). Figure 10(c) and

349 Figure 10(d) show similarities in flow structure to that of the single phase results in Figure

350 9.In super critical flow observed in Figure 10(a) and Figure 10(b), the normalized velocity

351 and normalized vorticity at the exit of the turbine in the downstream direction indicate that a

352 sufficient amount of interaction between the wake and the free surface is occurring. The

353 dissipation of energy that is occurring is affecting the pressure distribution about the turbine

354 blades and, thus, decreasing the torque being applied on the turbine blades.

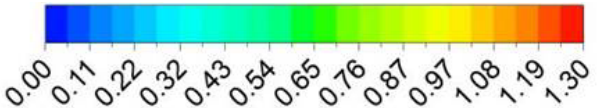

(a)

355

356

357

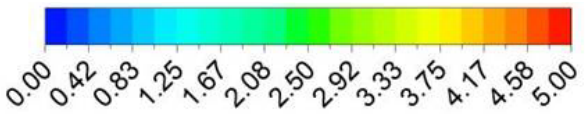

\section{(b)}

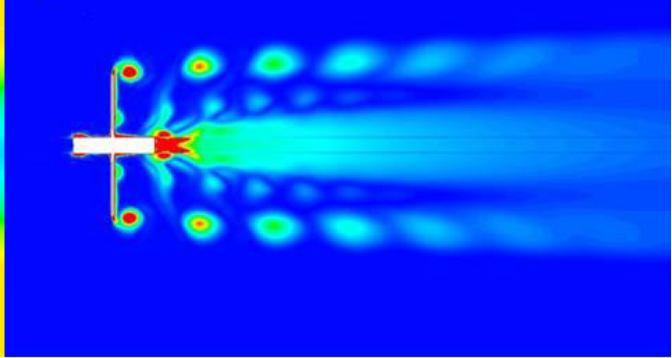

Figure 9. Normalized (a) velocity and (b) vorticity contours along the vertical meridional for single phase turbine operation in an infinite medium at a tip speed ratio of 1.86. 


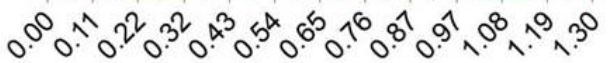

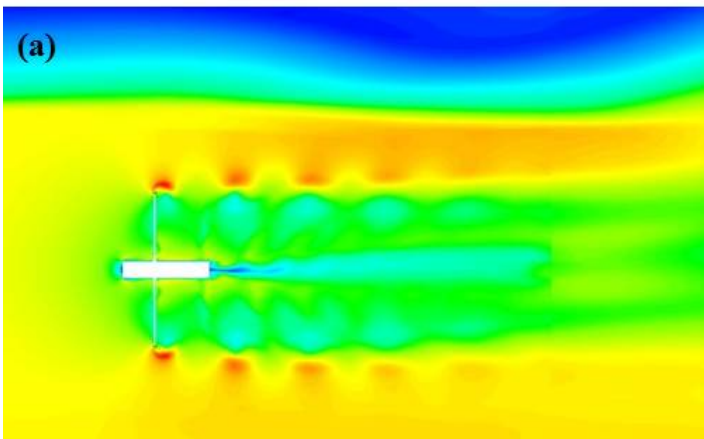

(c)

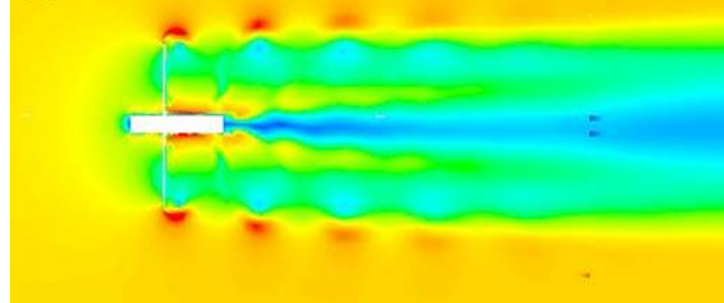

Figure 10. Normalized (a, c) velocityand (b,d) vorticitycontours along the vertical meridional at Froude numbers of (a, b) 1.31 and (c, d) 0.92 at a tip speed ratio of 1.86.

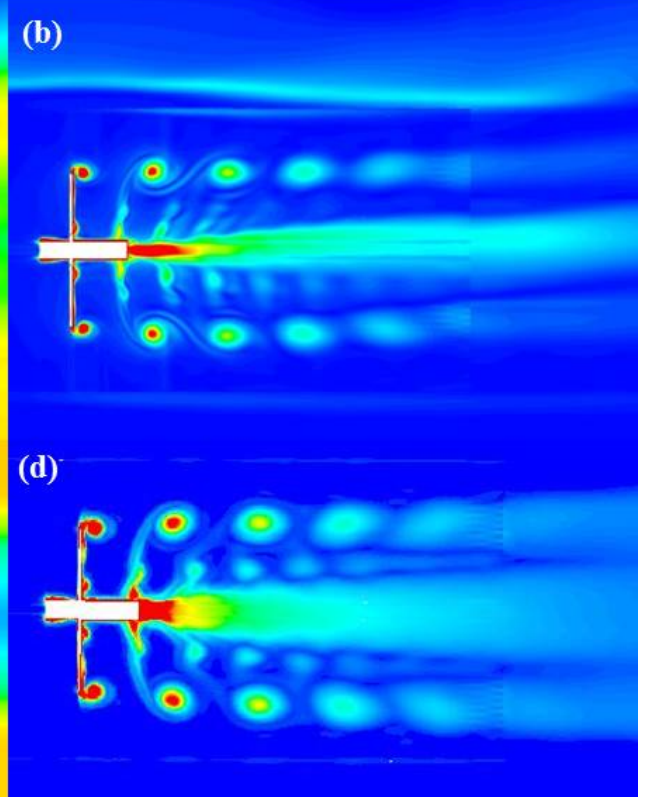

In Figure 11, the isosurfaces of the free surface with the corresponding contours of deviation from initial free surface level shown for Froude numbers of 1.31 and 0.92 . Comparing Figure 11 to Figure 10, less interaction between the free surface and the turbine is observed at lower values of Froude number. Both Figure 11(a) and Figure 11(b) show similar profiles near the turbine locations: primarily a rise in free surface elevation above the turbine operating position, a relative drop in free surface level immediately following the turbine, and diagonally oriented free surface levels downstream from the turbine. The diagonal free surface elevations observed in Figure 11(a) and (b) were also observed in the results obtained by Kolekaret al. (2015). The ripples observed in the downstream directions are a numerical artefact resulting from the meshing used. A significantly larger mesh would be needed to minimize the artificial ripples; however, such a mesh would not be economical given the primary outcome of the present simulation is the turbine performance coefficients. 


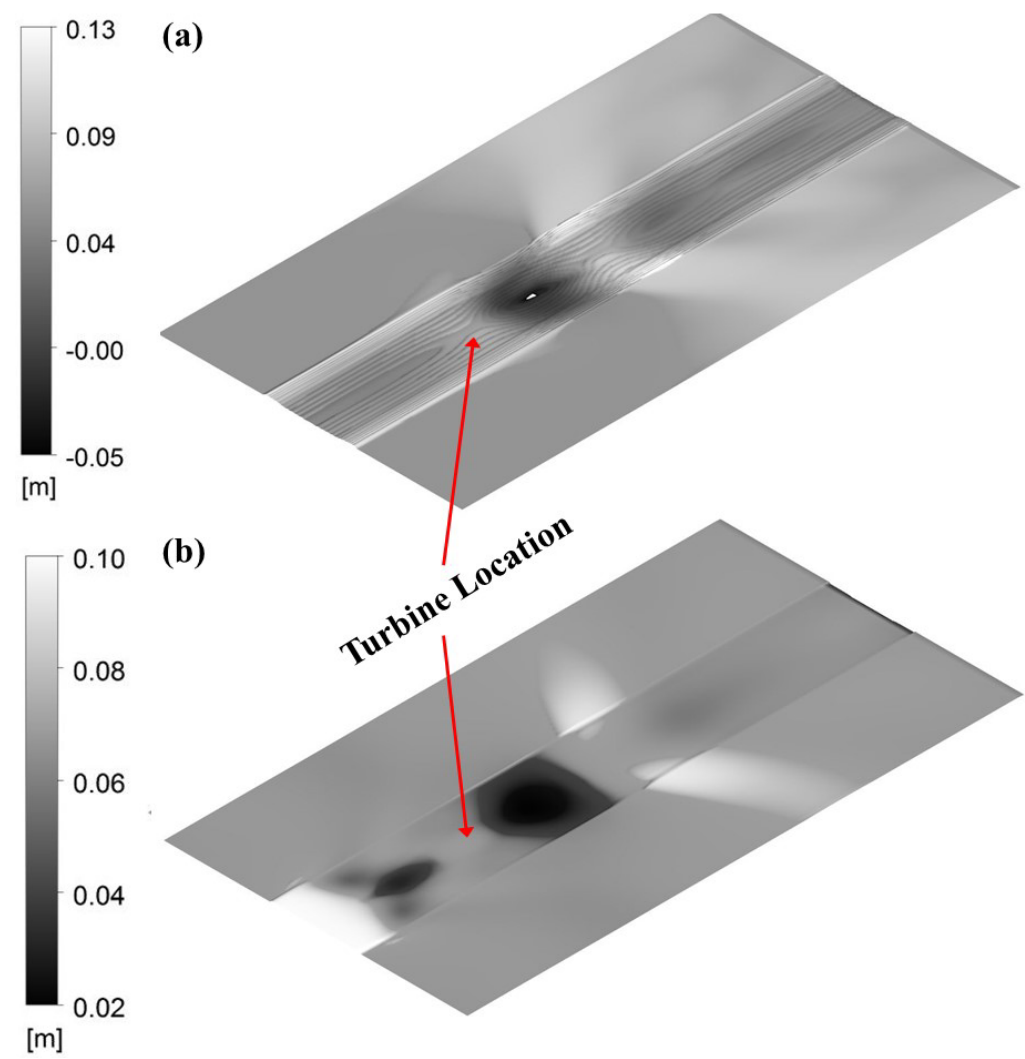

Figure 11. Isosurface of the free surface for cases of (a) $\mathrm{Fr}=1.31$ and (b) $\mathrm{Fr}=0.92$.

The linear lines positioned in the fluid geometry within Figure 12are used to analyse

wake data for each individual Froude number investigated as well as the single phase

simulation results. The profiles of streamwise component of the velocity in the three wake

positions downstream from the turbineare depicted in Figure 13 and Figure 14. Wake profiles areplotted at downstream locations of $D_{t}, 3 D_{t}$, and $5 D_{t}$, as illustrated in Figure 12. In each case, the turbine was positioned at the origin and the spanwise directionis normalized based on the turbine diameter. In Figure 13, the transition from super critical flow conditions to subcritical flow conditions may be observed. The flow transition occurs from Figure 13(b) where the Froude number is 1.04 to Figure 13(c) when the Froude number is reduced to a value of 0.92.Despite only a small change in the Froude number, wake profiles observed

386 between both cases exhibit vastly different characteristics. This proves that flow transition 387 occurs for a value of $F r 0.92 \leq \mathrm{Fr} \leq 1.04$. At approximately $\pm 0.5 \mathrm{Y} / D_{t}$, where tip vortices exist, the normalized velocity exhibits a local minimum at one diameter downstream.At this 
391

392

393

394

395

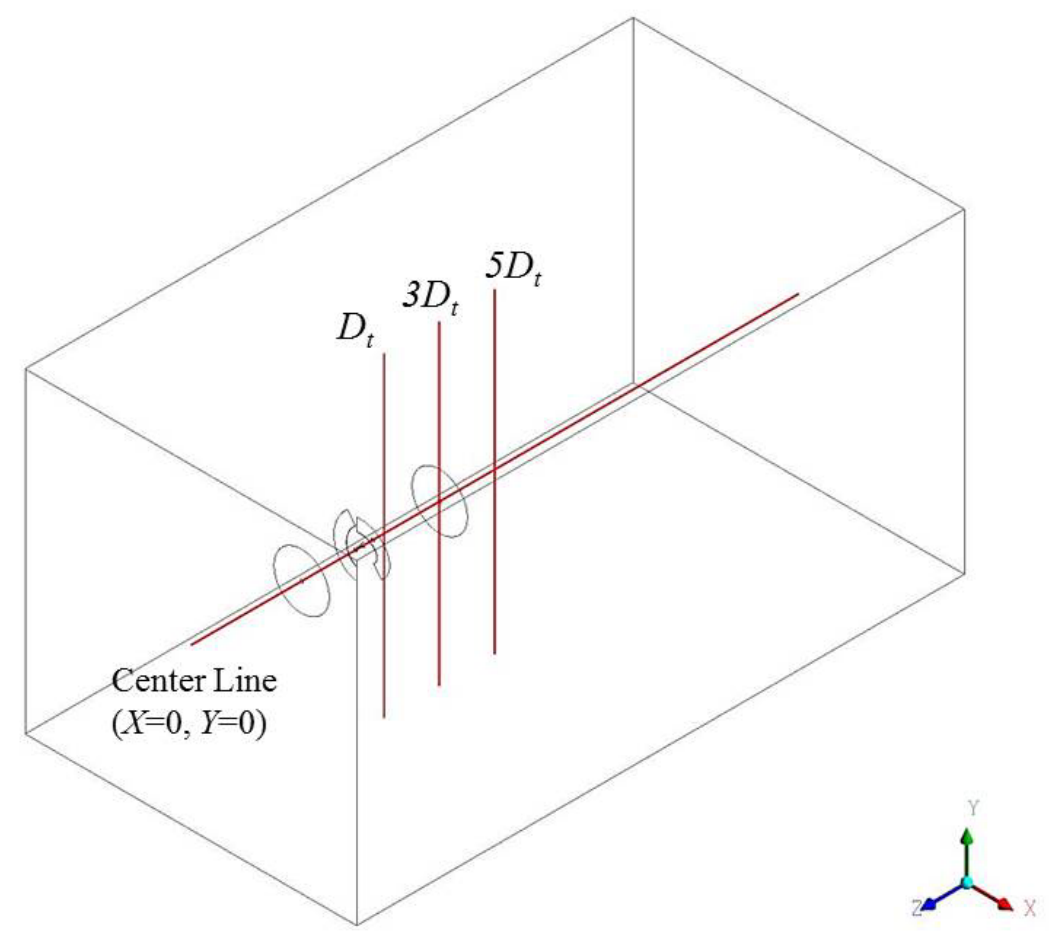

Figure 12. Linear geometric positioning for wake data acquisition and analysis.

Figure 14 compares the single phase wake profiles to the multiphase wake profiles at a Froude number of 0.71. Between Figure 14(a) and Figure 14(b), only marginal changes within the wake at each downstream distance are experienced. Therefore, as Froude number approaches zero, the wake profile slowly trends toward the profile observed during turbine operation in a single phase, infinite domain. In Figure 15, the normalized streamwise component of the velocity is displayed as a function the flow direction along the centreline.The axial coordinatewas normalized in the same manner as vertical coordinates fromFigure 13 andFigure 14. Positive values of $Z / D_{t}$ denote the upstream of the turbine while the negative values denote the downstream of the turbine. Each wake modelled had a normalized velocity of one at the domain inlet and recovered, at a minimum, $80 \%$ of that value at the exit. Each profile yields a sudden drop in velocity at the upstream side of the turbine, located at a normalized distance of zero. The single phase wake serves as the base case. As Froude number exceeded 1.0, an increase in normalized velocity magnitude from a 
minima of 0 to 0.25 along the central wake was experienced within $4 D_{t}$ downstream of the

407 turbine. The surge in the velocity minimaresulted from free surface interaction with the

408 turbine wake as theFroude number increased in value. The slow transition of this amplified

409 velocity can be observed from the peak values at corresponding Froude numbers of 0.92,

4101.04 , and 1.31. At a Froude number of 0.92 , a local normalized velocity maxima of 0.28

411 occurs three diameters downstream. When the Froude number was increased to a value of

4121.31 , the local normalized velocity maxima increased to a value of 0.75 located

413 approximately 3.5 diameters downstream.
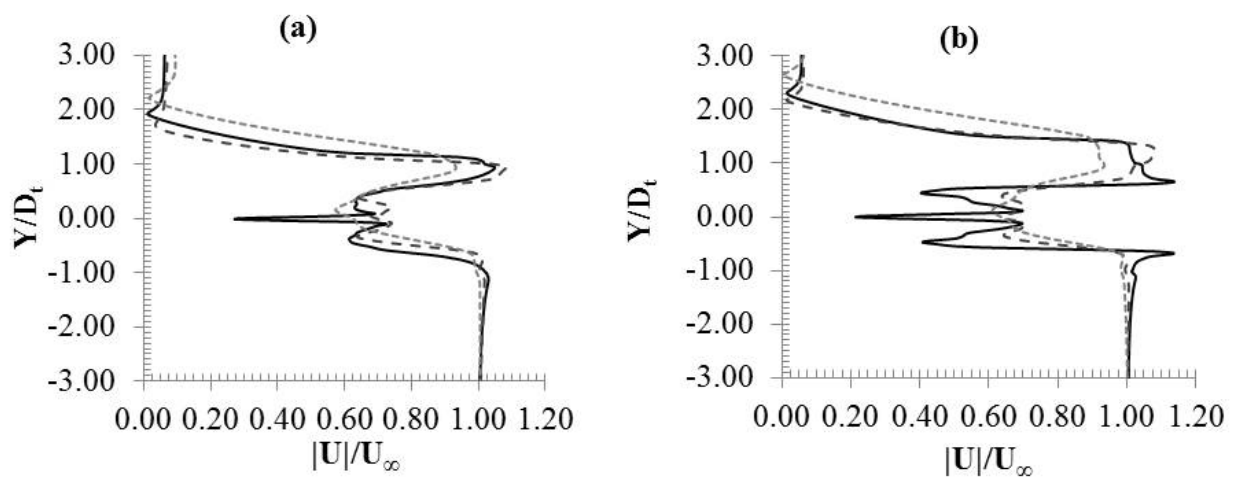

(c)

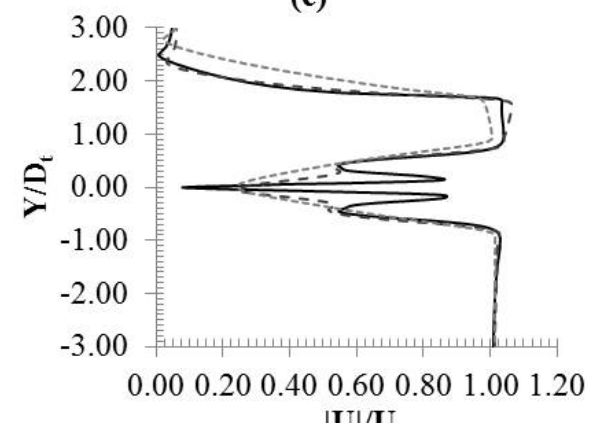

(d)

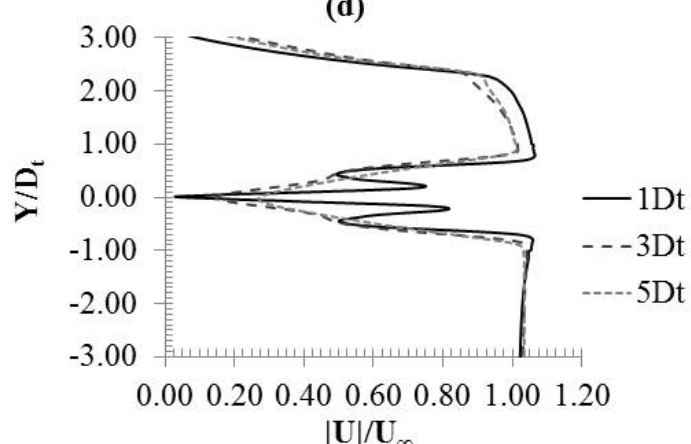

414

$|\mathbf{U}| / \mathbf{U}_{\infty}$

$|\mathbf{U}| / \mathbf{U}_{\infty}$

415 Figure 13. The profiles of the normalized streamwise component of the velocity for Fr of (a)

$416 \quad 1.31$, (b) 1.04 , (c) 0.92 , and (d) 0.71 at various locations downstream of the turbine. 
(a)

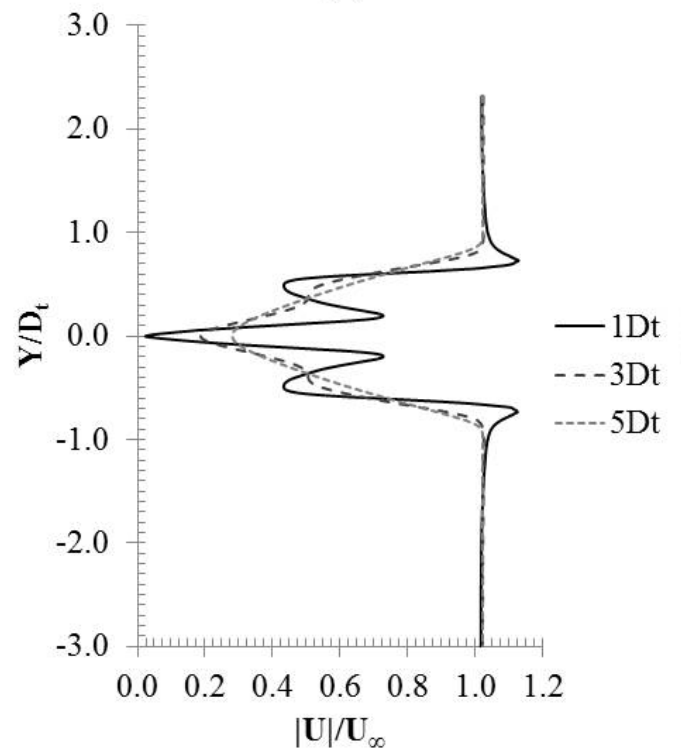

(b)

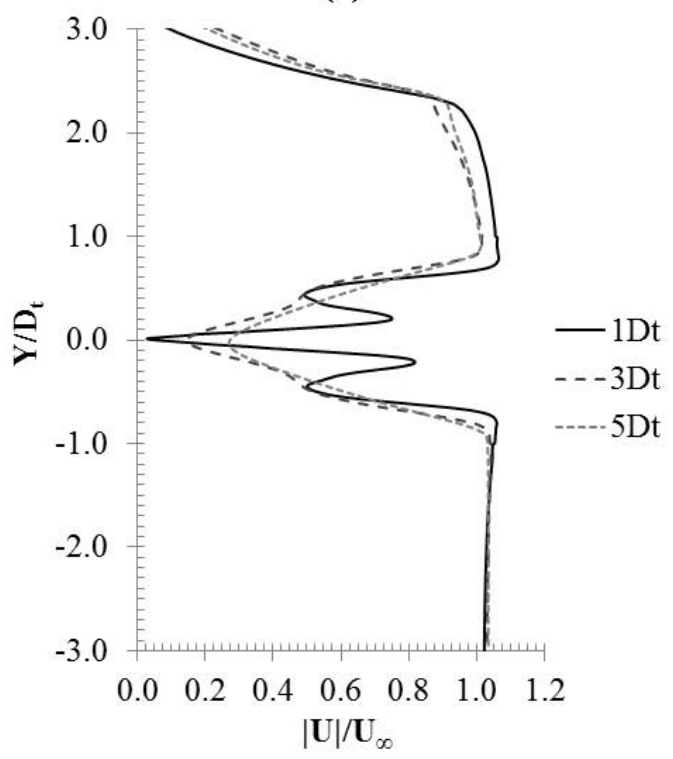

419

420

421

422

423

424

425

Figure 14. The profiles of the normalized streamwise component of the velocity for (a) single phase and (b) multiphase simulations at $\mathrm{Fr}=0.71$ at various locations downstream of the turbine.

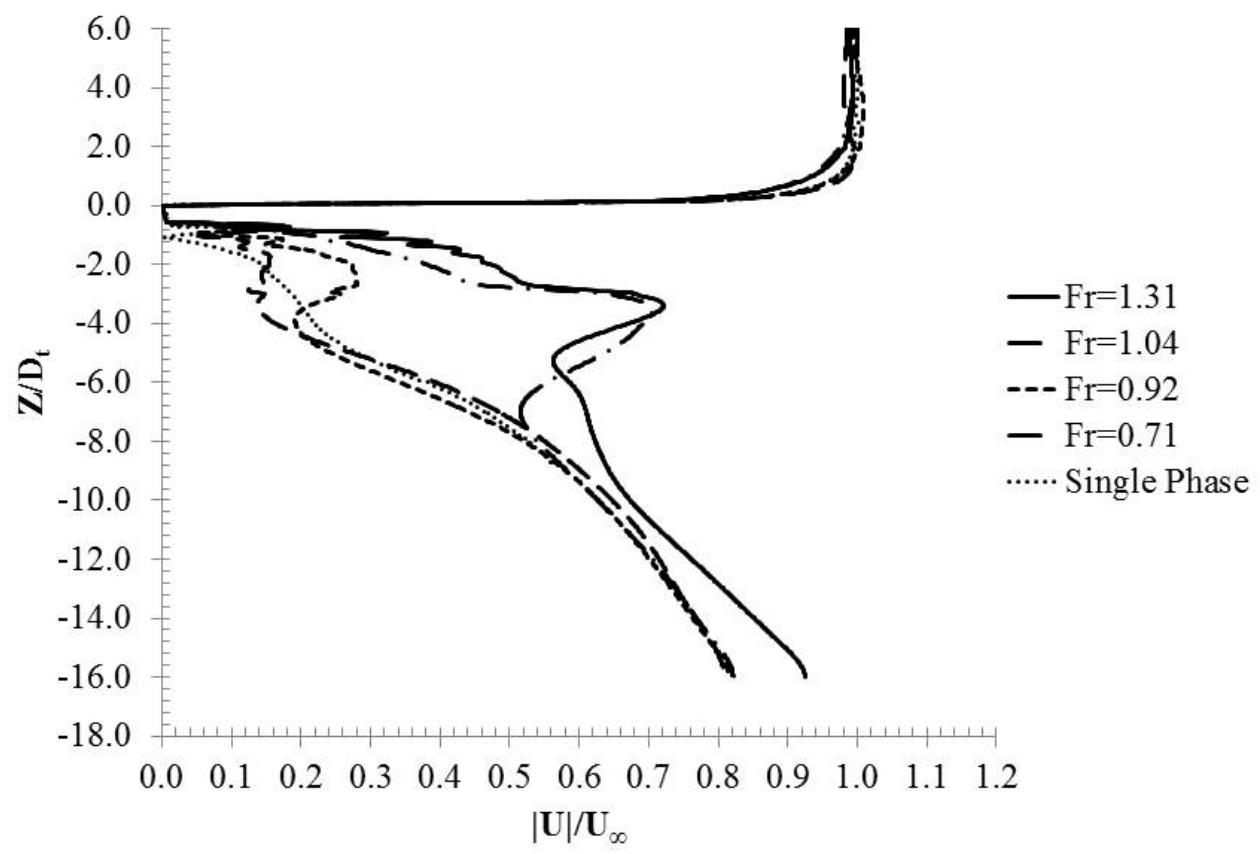

Figure 15. The profiles of the normalized streamwise component of the velocity along the flow direction for multiphase simulations at various values of $\mathrm{Fr}$ and for a single phase simulation. 


\section{Conclusions}

The VOF multiphase model was used to capture transient effects resulting from hydrokinetic turbine operation near the free surface. Source dampening was applied at the inlet of the domain to provide additional numerical stability. The method applied was validated by simulating flow over a rectangular cylinder positioned near the free surface and compared to corresponding results provided by Malavasi and Guadagnini (2007). Based on the coefficient of drag, coefficient of lift, and Strouhal number, the maximum error between numerical and experimental results was approximately $6.1 \%$.

Performance characteristics of the turbine were mapped as a function of both the Froude number and the tip speed ratio. Single phase results of the propeller-based turbine resulted in power coefficient and thrust coefficient values of approximately 0.44 and 1.20, respectively(Schleicher et al.,2015). Predicted power coefficient and thrust coefficient values determined from multiphase simulations were within $5 \%$ of those predicted by the single phase simulations. Results obtained by Bai et al. (2014) show similar trends in performance at subcritical flow conditions. Multiphase results show that when the flow becomes critical, meaning Froude number is equal to or greater than a value of one, a sharp decrease in mechanical power is experienced by the unit. Froude numbers of 1.04 and 1.31 resulted in an average $32.2 \%$ drop in power from results obtained in subcritical conditions.

Wake profiles of the normalized streamwise component of the velocity manifest the presence of the flow transition as $F r$ exceeds unity. A significant shift in velocity distributions occurs between Froude numbers of 0.92 and 1.04, highlighting the shift from subcritical to supercritical flow. During supercritical flow, the wake 
450 within four to five diameters downstream interacted with the free surface and shifted 451 upwards.

452 Acknowledgements

453 The authors would like to gratefully acknowledge funding from the Office of Naval 454 Research. The authors are also thankful for Bob Klein and e-Harvest for continued support 455 and collaboration.

\section{$456 \quad$ Funding}

This project was funded by the Office of Naval Research under Award N00014-12-

$458 \mathrm{M}-0050$ through a SBIR project with e-Harvest.

459 


\begin{tabular}{|c|c|c|c|}
\hline$A$ & area, $\mathrm{m}^{2}$ & & Greek Symbols \\
\hline$B$ & channel width, $\mathrm{m}$ & $\alpha, b, \sigma$ & closure coefficients \\
\hline C & coefficient & $\alpha$ & volume fraction \\
\hline $\mathrm{Cu}$ & Courant number & B & relative angle, ${ }^{\circ}$ \\
\hline c & damping resistance, $1 / \mathrm{m}$ & $\Delta$ & change in variable \\
\hline$D$ & diameter, m & $\lambda$ & tip speed ratio \\
\hline$d$ & depth, m & $\vartheta$ & wrap angle, ${ }^{\circ}$ \\
\hline$f$ & frequency, $1 / \mathrm{s}$ & $\mu$ & dynamic viscosity, Pa-s \\
\hline$F$ & blending function & $v$ & kinematic viscosity, $\mathrm{m}^{2} / \mathrm{s}$ \\
\hline$F$ & forces, $\mathrm{N}$ & $\varphi$ & sink term, kg/s \\
\hline $\mathrm{Fr}$ & Froude number & $\rho$ & density, $\mathrm{kg} / \mathrm{m}^{3}$ \\
\hline$g$ & gravity, $\mathrm{m} / \mathrm{s}^{2}$ & $\sigma$ & solidity \\
\hline$g$ & damping function & $\tau$ & stress tensor, $\mathrm{Pa}$ \\
\hline$h$ & water depth, m & $\omega$ & angular velocity, $1 / \mathrm{s}$ \\
\hline$k$ & turbulent kinetic energy, $\mathrm{J} / \mathrm{kg}$ & $\widetilde{\omega}$ & normalized vorticity \\
\hline I & cylinder cross section length, m & & \\
\hline$M$ & mass source, $\mathrm{kg} / \mathrm{s}$ & & Subscripts \\
\hline$m$ & meridional length, $\mathrm{m}$ & $a$ & absolute \\
\hline$\dot{m}$ & mass flow, $\mathrm{kg} / \mathrm{s}$ & $B$ & blades \\
\hline$P$ & pressure, $\mathrm{Pa}$ & $b$ & bottom \\
\hline $\operatorname{Re}$ & Reynolds number & $c$ & cell \\
\hline$r$ & refinement factor & $D$ & drag \\
\hline$S$ & mean rate-of-strain tensor, $1 / \mathrm{s}$ & $e$ & end \\
\hline St & Strouhal number & $f$ & cell face \\
\hline$s$ & cylinder cross section width, m & fs & free surface level \\
\hline$t$ & time, $\mathrm{s}$ & $h$ & at the hub \\
\hline$T$ & thrust, $\mathrm{N}$ & $i, j, k, l, s, t$ & tensor indices \\
\hline$U$ & velocity, $\mathrm{m} / \mathrm{s}$ & $L$ & lift \\
\hline$\widetilde{U}$ & normalized velocity & $m$ & at the mean value \\
\hline$V$ & volume, $\mathrm{m}^{3}$ & $\max$ & maximum value \\
\hline$\dot{W}$ & power, W & $p$ & secondary phase \\
\hline$X$ & distance in $\mathrm{x}$-direction, $\mathrm{m}$ & $q$ & primary phase \\
\hline$Y$ & distance in $\mathrm{y}$-direction, $\mathrm{m}$ & $s$ & start \\
\hline$Z$ & distance in $\mathrm{z}$-direction, $\mathrm{m}$ & $T$ & turbulent / thrust \\
\hline \multirow[t]{3}{*}{$Z_{B}$} & quantity & $t$ & at the tip \\
\hline & & $\infty$ & freestream \\
\hline & Superscript & & \\
\hline * & $\begin{array}{l}\text { closure coefficient/non- } \\
\text { dimensional }\end{array}$ & & \\
\hline , & denotes the blade angle & & \\
\hline
\end{tabular}




\section{References}

Bahaj, A. S., Molland, A. F., Chaplin, J. R., \& Batten, W. M. (2007). Power and Thrust Measurements of Marine Current Turbines Under Various Hydrodynamic Flow Conditions in a Cavitation Tunnel and a Towing Tank. Renewable Energy, 32, 407426.

Bai, X., Avital, E. J., Munjiza, A., \& Williams, J. J. (2014). Numerical Simulation of a Marine Current Turbine in Free Surface Flow. Renewable Energy, 63, 715-723.

Cebrián, D., Ortega-Casanova, J., \& Fermandez-Feria, R. (2013). Lift and Drag Characteristics of a Cascade of Flat Plates in a Configuration of Interest for a Tidal Current Energy Converter: Numerical Simulations Analysis. Journal of Renewable and Sustainable Energy, 5, 1-19.

Hirt, C. W., \& Nichols, B. D. (1981). Volume of Fluid (VOF) Method for the Dynamics of Free Boundaries. Journal of Computational Physics, 39(1), 201-225.

Kolekar, N., \& Banerjee, A. (2015). Performance Characterization and Placement of a Marine Hydrokinetic Turbine in a Tidal Channel Under Boundary Proximity and Blockage Effects. Applied Energy, 148, 121-133.

Kolekar, N., Hu, Z., Banerjee, A., \& Du, X. (2013). Hydrodynamic Design and Optimization of Hydro-kinetic Turbines using a Robust Design Method. Proceedings of the 1st Marine Energy Technology Symposium. Washington, D.C.

Kolekar, N., Mukherji, S. S., \& Banerjee, A. (2011). Numerical Modeling and Optimization of Hydrokinetic Turbine. ASME 2011 5th International Conference on Energy Sustainability. Washington, D.C.

Liu, I., Riglin, J., Schleicher, W., \& Oztekin, A. (2015). Flow Past a Plate in the Vicinity of a Free Surface. Ocean Engineering, Under Review.

Malavasi, S., \& Guadagnini, A. (2007). Interactions Between a Rectangular Cylinder and a Free-Surface Flow. Journal of Fluids and Structures, 23(8), 1137-1148.

Menter, F. R. (1993). Zonal Two-Equation k- $\omega$ Turbulence Models for Aerodynamic Flows. AIAA Paper 93-2906.

Menter, F. R. (1994). Two-Equation Eddy-Viscosity Turbulence Models for Engineering Applications. AIAA Journal, 32(8), 1598-1605.

Mukherji, S. S., Kolekar, N., Banerjee, A., \& Mishra, R. (2011). Numerical Investigation and Evaluation of Optimum Hydrodynamic Performance of a Horizontal Axis Hydrokinetic Turbine. Journal of Renewable and Sustainable Energy, 3, 1-18.

Noh, W. F., \& Woodward, P. (1976). SLIC (Simple Line Interface Calculation). In A. I. Vooren, \& P. J. Zandergen (Ed.), 5th International Conferenceof Fluid Dynamics, 59, pp. 330-340. doi:10.1007/3-540-08004-x_336 
Park, J. C., Kim, M. H., \& Miyata, H. (1999). Fully Non-linear Free-surface Simulations By a 3D Viscous Numerical Wave Tank. International Journal for Numerical Methods in Fluids, 29, 685-703.

Reichl, P., Hourigan, K., \& Thompson, M. C. (2005). Flow Past a Cylinder Close to a Free Surface. Journal of Fluid Mechanics, 533, 269-296.

Riglin, J. D., Schleicher, W. C., Kraybill, Z., Klein, R. C., \& Oztekin, A. (2013). Computational Fluid Dynamics and Structural Finite Element Analysis of a Micro Hydro Turbine. ASME 2013 International Mechanical Engineering Congress and Exposition. San Diego, CA.

Riglin, J., Schleicher, W. C., \& Oztekin, A. (2015, March 12). Numerical Analysis of a Shrouded Micro-Hydrokinetic Turbine Unit. Journal of Hydraulic Research. doi:Accepted

Riglin, J., Schleicher, W., \& Oztekin, A. (2014). Diffuser Optimization of a MicroHydrokinetic Turbine. ASME 2014 International Mechanical Engineering Congress and Exposition. Montreal, Canada.

Roache, P. J. (1993). A Method for Uniform Reporting of Grid Refinement Studies. Proceedings of the 11th AIAA Computational Fluid Dynamics Conference. Orlando.

Schleicher, W. C., Riglin, J. D., Kraybill, Z. A., \& Oztekin, A. (2013). Design and Simulaiton of a Micro Hydrokinetic Turbine. 1st Marine Energy Technology Symposium. Washington, D.C.

Schleicher, W., Ma, H., Riglin, J., Kraybill, Z., Wei, W., Klein, R., \& Oztekin, A. (2014). Characteristics of a Micro-Hydro Turbine. Journal of Renewable and Sustainable Energy, 6, 1-14.

Schleicher, W., Riglin, J., \& Oztekin, A. (2015). Numerical Characterization of a Preliminary Portable Micro-hydrokinetic Turbine Rotor Design. Renewable Energy, 76, 234-241.

Schleicher, W., Riglin, J., Oztekin, A., \& Klein, R. (2014). Numerical Optimization of a Portible Hydrokinetic Turbine. 2nd Marine Energy Technology Symposium. Seattle, WA.

U.S. Geological Survey. (2014). (National Water Information System data available on the World Wide Web (Water Data for the Nation)) Retrieved January 9, 2014, from http://waterdata.usgs.gov/nwis/

Whelan, J., Graham, J., \& Peiro, J. (2009). A Free-Surface and Blockage Correction for Tidal Turbines. Journal of Fluid Mechanics, 624, 281-291.

Wilcox, D. C. (2010). Turbulence Modeling for CFD. La Canada, California: DCW Industries, Inc. 
534 Zwart, P. J., Godin, P. G., Penrose, J., \& Rhee, S. H. (2007). Ship Hull Simulations with a

535 Coupled Solution Algorithm. Proceedings of the 10th International Symposium on Practical Designs of Ships and Other Floating Structures. Houston, TX.

537

538 